\title{
CaracterizaÇão de CONSUMo e dePendênCIA de TABACo ENTRE TRABALHADORES DE UMA INSTITUIÇÃO DE NÍVEL SUPERIOR ${ }^{1}$
}

\author{
Edilaine Cristina da Silva Gherardi-Donato ${ }^{2}$; Miriam Lopes ${ }^{3}$; \\ Mariana Rodrigues Santiago ${ }^{4}$; Clarissa Mendonça Corradi-Webster ${ }^{5}$
}

\begin{abstract}
Trata-se de estudo quantitativo de corte transversal, cujo objetivo foi caracterizar o perfil de consumo e dependência de tabaco entre funcionários não docentes de uma universidade pública, realizado no período de fevereiro a agosto de 2008. Utilizou-se questionário autoaplicável, composto por dados sociodemográficos, dados sobre tabagismo e questionário de Fagerström. A amostra foi constituída por 149 funcionários. Os resultados indicam prevalência do consumo de tabaco de $12,1 \%$ dos entrevistados, sendo que $88,8 \%$ dos fumantes anseiam por parar de fumar, mas poucos conseguem sem ajuda adequada, o que demonstra a necessidade de oferecer apoio formal para o sucesso desse anseio a esses trabalhadores, assim como a inserção de ações educativas de prevenção nessa temática.
\end{abstract}

Descritores: Enfermagem; Transtornos Relacionados ao Uso de Substâncias; Tabaco; Trabalho.

\footnotetext{
${ }^{1}$ Apoio financeiro da Fundação de Amparo à Pesquisa do Estado de São Paulo (FAPESP), processo nº 2009/004575.

${ }^{2}$ Enfermeira, Doutor em Enfermagem, Professor Doutor, Escola de Enfermagem de Ribeirão Preto, Universidade de São Paulo, Centro Colaborador da OMS para o Desenvolvimento da Pesquisa em Enfermagem, SP, Brasil. E-mail: nane@eerp.usp.br.

${ }^{3}$ Enfermeira, Mestranda em Enfermagem, Escola de Enfermagem de Ribeirão Preto, Universidade de São Paulo, Centro Colaborador da OMS para o Desenvolvimento da Pesquisa em Enfermagem, SP, Brasil. Bolsista do Conselho Nacional de Desenvolvimento Científico e Tecnológico (CNPq). E-mail: mi.lops@hotmail.com.

${ }^{4}$ Enfermeira, Mestranda em Enfermagem, Escola de Enfermagem de Ribeirão Preto, Universidade de São Paulo, Centro Colaborador da OMS para o Desenvolvimento da Pesquisa em Enfermagem, SP, Brasil. E-mail: santinhago@hotmail.com.

${ }_{5}^{5}$ Psicóloga, Doutor em Psicologia, Professor Doutor, Departamento de Psicologia, Faculdade de Filosofia, Ciências e Letras de Ribeirão Preto, Universidade de São Paulo, SP, Brasil. E-mail: clarissac@usp.br.
}

Endereço para Correspondência 


\section{Characterization of tobacco Consumption AND DePendence among WORKERS AT A HIGHER EDUCATION INSTITUTION}

Cross-sectional quantitative study to characterize the profile of tobacco consumption and dependence among non-teaching staff at a public university, conducted between February and August 2008. A self-administered questionnaire was used, comprising socio-demographic data, data on smoking and the Fagerström Questionnaire. The sample consisted of 149 employees. The results indicate the prevalence of smoking in $12.1 \%$ of respondents; $88.8 \%$ of smokers wish to quit smoking, but few of them are able to without appropriate support, which demonstrates the need to offer formal support for these workers to successfully achieve quitting. Also, educative prevention actions in this area need to be set up.

Descriptors: Nursing; Substance-Related Disorders; Tobacco; Work.

\section{Caracterización del CONSUMO Y DEPENDEnCIA DEL TABACO ENTRE TRABAJADORES EN UNA INSTITUCIÓN DE NIVEL SUPERIOR}

Estudio cuantitativo de corte transversal que tuvo como objetivo caracterizar el perfil de consumo y dependencia al tabaco entre el personal no docente de una universidad pública, realizado entre febrero y agosto de 2008. Se utilizó un cuestionario autoadministrado que incluye datos socio-demográficos, datos sobre el tabaquismo y el Cuestionario de Fagerström. La muestra comprendió a 149 empleados. Los resultados indican la prevalencia de tabaquismo en el $12.1 \%$ de los encuestados, con el $88.8 \%$ de los fumadores que anhelan dejar de fumar, pero muy pocos consiguen sin el apoyo adecuado, lo que demuestra la necesidad de ofrecer apoyo formal al éxito de este anhelo entre estos trabajadores, así como la necesidad de incluir acciones educativas de prevención en este ámbito.

Descriptores: Enfermería; Trastornos Relacionados con Sustancias; Tabaco; Trabajo.

\section{Introdução}

O tabaco é uma das drogas lícitas mais utilizadas, sendo o seu consumo um dos maiores problemas de saúde pública, tanto nacional quanto internacionalmente. Sua planta, de origem americana, chegou à Europa no século XVI e seu consumo já esteve associado a comportamentos sociais que representavam do glamour à rebeldia ${ }^{(1)}$.

Um bilhão e 200 milhões de pessoas, atualmente, fumam no mundo ${ }^{(2-4)}$, sendo a prevalência de $25 \%$ na população adulta norte-americana, aproximadamente a mesma prevalência encontrada em estudo realizado em São Paulo, que evidenciou prevalência de $24 \%{ }^{(2)}$.
Segundo estimativas do Instituto Nacional do Câncer (Inca), no Brasil ocorrem 200.000 mortes/ano associadas ao tabagismo, ou seja, 23 pessoas morrem a cada hora pelo uso do tabaco ${ }^{(5)}$.

A idade média para início do consumo de tabaco está entre 13 e 14 anos, porém, a vulnerabilidade para dependência não está relacionada apenas à idade, mas, também, a outros aspectos como uso do tabaco pelos pais, colegas mais velhos e influência da mídia, esses são considerados fatores preditores de seu consumo. Já a expectativa de vida de um indivíduo que fuma 
grande quantidade de cigarros é $25 \%$ menor que a de um não fumante ${ }^{(1)}$. E, entre as 25 doenças causadoras de morte, relacionadas ao hábito de fumar, tem-se: doenças cardiovasculares (43\%), câncer (36\%), doenças respiratórias $(20 \%)$ e outras $(1 \%)^{(2)}$.

A nicotina, componente psicoativo do tabaco, apresenta propriedades de reforço positivo e de adição, as quais contribuem para os efeitos estimulantes básicos da nicotina no sistema nervoso central. Em termos comportamentais, esses efeitos estimulantes da nicotina resultam em melhor atenção, aprendizagem, tempo de reação e capacidade para solucionar problemas, assim como o hábito de fumar melhora o humor e diminui a tensão. Porém, seus efeitos adversos, devido à toxicidade dessa substância incluem náuseas, vômito, palidez, tontura, cefaleia, aumento da pressão sanguínea e taquicardia, entre outros $^{(6)}$.

Em pessoas cujo consumo de tabaco se faz em longo prazo, a ausência da nicotina no organismo pode provocar algumas características como tensão, dificuldade para concentrar-se, sonolência, problemas para dormir, redução da pressão sanguínea, ganho de peso, desempenho motor diminuído e maior tensão muscular ${ }^{(6)}$.

Estudo realizado na Universidade Federal de São Paulo demonstrou que a prevalência de uso de tabaco foi maior entre funcionários não docentes quando se comparou a estudantes e docentes da instituição. Desde a Primeira Guerra Mundial, iniciou-se aumento significativo de consumo de tabaco, sendo que o consumo per capita cresceu à taxa de 5 a $15 \%$ ao ano, durante a primeira metade do século $\mathrm{XX}^{(7)}$.

De acordo com os autores, no Brasil, o consumo de cigarros cresceu até 7\% durante a década de 70, mas, a partir de 1980, esse ritmo reduziu o consumo per capita para níveis de $1 \%$ ao ano, na população com 15 anos ou mais de idade. Contudo, entre 1970 e 1990, enquanto a população cresceu $61,5 \%$, o consumo de cigarros aumentou $125,7 \%$. Através de levantamento feito pelo IBGE, em 1989, estimou-se em quase 31 milhões o número de tabagistas, representando $32,6 \%$ da população acima dos 15 anos. Na faixa etária entre 15 e 19 anos temse 2,4 milhões de fumantes, sendo que $75 \%$ dos fumantes iniciam o consumo de tabaco entre 10 e 18 anos de idade. Embora sejam necessárias políticas abrangentes e rigorosas, as campanhas antitabagismo têm influenciado o comportamento dos fumantes e o alvo prioritário de ação antifumo em países subdesenvolvidos, conforme considera a Organização Mundial de Saúde (OMS), deve ser centrado nos profissionais de saúde.

Destacou-se, em estudo realizado com trabalhadores de empresas no Rio de Janeiro, que, entre os possíveis riscos para o uso de tabaco, se encontram o estresse no trabalho, a rivalidade com os colegas e a insatisfação com o trabalho e com as funções que desempenham. A sugestão dos pesquisadores é a execução de estratégias preventivas no ambiente de trabalho, a conscientização sobre o consumo de substâncias e o incentivo à prática de esportes $^{(8)}$.

Diante dos estudos e das solicitações frequentes dos trabalhadores, por informações sobre o uso de tabaco, considerou-se desenvolver pesquisa sobre o consumo e a dependência de tabaco entre trabalhadores de uma universidade pública do interior paulista, cujo objetivo foi caracterizar o perfil de consumo e dependência de tabaco entre funcionários não docentes de uma universidade pública, através do levantamento de informações sobre o padrão de consumo de tabaco e a caracterização sociodemográfica dessa população.

\section{Material e Métodos}

Tipo de estudo

Trata-se de estudo quantitativo, descritivo e exploratório, de corte transversal, cujo intuito foi observar, descrever e explorar aspectos de uma situação de pesquisa.

Local, amostra e periodo

Realizou-se o estudo com os trabalhadores não docentes de uma unidade de ensino superior localizada em um campus universitário do interior paulista, a qual possui 14 departamentos, acrescida das seções administrativa, acadêmica e financeira. A escolha dessa unidade para a coleta de dados ocorreu por essa possuir maior número de funcionários dentre as demais unidades do campus, além de possuir maior variabilidade de funções entre outras características sociodemográficas.

A unidade possui população de 488 servidores na categoria não docente. Considerando apenas os departamentos selecionados para a coleta pelos entrevistadores, obteve-se 355 servidores. Desses, 149 (41,94\%) concordaram em participar da pesquisa, respondendo os questionários. Do total de 355, 70 funcionários não quiseram participar e 136 não foram localizados, ou seja, funcionários que estavam de férias, mudaram de unidade ou se desligaram da instituição. Dessa forma, o critério de inclusão na pesquisa foi ser funcionário não docente da unidade. Os dados foram coletados nos meses de fevereiro a agosto de 2008 .

\section{Coleta de dados}

A coleta de dados foi realizada com o uso de questionário composto por questões de identificação (idade, sexo, com quem mora, estado civil, se tem filhos, renda familiar, número de pessoas que residem na casa, grau de escolaridade, religião, ocupação, função, tempo de trabalho na instituição) $)^{(9)}$ e informações relacionadas ao consumo de tabaco, onde foram inclusas questões sobre o hábito de fumar e a dependência de tabaco.

Para o rastreamento da intensidade da dependência ao tabaco foram utilizadas as questões do Fagerström Test for Nicotine Dependence (FTNQ, questionário de dependência nicotínica de Fagerström), composto por 6 itens, os quais apresentam diferentes pesos e cujo escore varia de 0 a $10^{(10)}$. Esse instrumento originouse do Fagerström Tolerance Questionnaire (FTQ, questionário de tolerância de Fagerström), elaborado em $1978^{(11)}$, constituído inicialmente por 8 questões, que, 
após ser revisto devido às significativas desvantagens psicométricas do $\mathrm{FTQ}^{(12)}$, reduziu-se para as 6 questões conhecidas atualmente, referentes ao hábito de fumar. Assim, sugeriu-se mudar a nomenclatura do teste para questionário de dependência nicotínica de Fagerström (FTND) ${ }^{(10)}$. Tal instrumento foi validado no Brasil, em 1991, por Carmo e Pueyo (2002) ${ }^{(13)}$. Ambos elaborados por Fagerström et al. ${ }^{(14-15)}$. No Brasil, esse instrumento vem sendo utilizado em diversos estudos ${ }^{(10,16-17)}$, inclusive acerca de suas propriedades psicométricas que sugerem o FTND como instrumento confiável para a avaliação de fumantes em diferentes populações ${ }^{(12)}$.

\section{Considerações eticas}

O projeto foi submetido e aprovado pelo Comitê de Ética em Pesquisa da Escola de Enfermagem de Ribeirão Preto (CEP), sob Protocolo n ${ }^{\circ} 0846 / 2007$.

Os participantes assinaram o termo de consentimento livre e esclarecido, respeitando-se os procedimentos da Resolução 196/96 ${ }^{(18)}$, do Conselho Nacional de Saúde.

\section{Análise dos dados}

As informações coletadas foram registradas e armazenadas em planilha de cálculo do Microsoft Excel 2007, para posterior migração em um banco de dados do programa estatístico Statistical Package Social Science (SPSS, versão 16.0, de 2007), onde foi realizada a análise estatística descritiva.

\section{Resultados}

A amostra foi composta por 149 sujeitos, que aceitaram participar da pesquisa. Dentre os participantes, $74,3 \%$ eram do sexo feminino, 59,2\% residiam com a família atual, 63,8\% eram casados ou amasiados, 64,5\% tinham filhos que moram consigo, $52,6 \%$ possuíam ensino superior completo, $62,5 \%$ pertenciam à religião católica e $48,7 \%$ ocupavam funções que exigem como escolaridade o nível médio. A média de idade da amostra foi de 44,3 anos $(\mathrm{d} p=8,54 ;$ mín=23, máx=61). A média da renda familiar encontrada foi de $\mathrm{R} \$ 4.238$ ( $\mathrm{dp}=2.231$; mín=800, máx=12.000).

Os resultados da aplicação do questionário sobre tabagismo evidenciaram que $29,5 \%$ de sujeitos já fumaram em algum momento de suas vidas; $12,1 \%$ fumam atualmente; $16,1 \%$ eram ex-fumantes; $8,7 \%$ já tiveram problemas decorrentes do uso de cigarro e 2,0\% disseram ter procurado ajuda para parar de fumar. Dos 13 sujeitos, que relataram ter tido problemas pelo uso de cigarro, foram identificados $69,2 \%$ que tiveram problemas físicos, $23,1 \%$ problemas sociais, e apenas $7,7 \%$ responderam ter sofrido problemas emocionais devido ao uso de cigarros. Os três sujeitos que procuraram ajuda para cessar o uso de tabaco relataram o uso de adesivos de nicotina, participação de terapias em grupo específica para parar de fumar e uso de medicação que auxilia na obtenção do objetivo em questão.

Com relação aos ex-fumantes, 16 entrevistados relataram que decidiram parar de fumar devido aos malefícios que o cigarro acarreta ou poderá acarretar em sua saúde, 5 pessoas disseram que o cigarro não significava nada em suas vidas e 4 relataram que pararam de fumar por motivos sociais, ou seja, por solicitação de filhos e cônjuges.

A aplicação do questionário sobre o hábito de fumar, incluindo as questões de dependência do questionário de Fagerström, aos 18 participantes que se consideraram fumantes atualmente, obteve-se que $61,1 \%$ relataram fumar o primeiro cigarro 60 minutos depois de acordar; $16,7 \%$ disseram encontrar dificuldades para evitar fumar em lugares onde é proibido; 61,1\% relataram que qualquer cigarro do dia é difícil de largar ou de não fumar; $61,1 \%$ responderam que fumam até 10 cigarros por dia; 33,3\% disseram fumar mais nas primeiras horas do dia do que durante o resto do dia; $38,9 \%$ fumavam mesmo estando doente e de cama; 88,8\% gostariam de parar de fumar; $50 \%$ não sabiam determinar quando gostariam de parar de fumar; $72,2 \%$ já tentaram parar de fumar, mas continuam. Os sujeitos que referiram querer parar de fumar (16) citaram motivos relacionados à saúde $(81,4 \%)$, ao convívio social $(12,4 \%)$ e ao estado psicológico (6,2\%). Entre os fatores que dificultam parar de fumar foram citados: hábito de fumar que possuem, vontade que sentem em fumar, ansiedade, nervosismo, falta de adaptação à medicação de apoio, problemas familiares, estado emocional e influência de amigos e ambientes sociais.

Entre os ex-fumantes (24), 91,6\% responderam que pararam de fumar de uma vez e apenas $8,4 \%$ disseram que foram diminuindo o número de cigarros até parar. Em relação ao tempo em que essas pessoas são ex-fumantes, $41,7 \%$ deixaram de fumar há 21-31 anos, 25\% encontravamse no período de 11 a 20 anos, 20,8\% permanecem sem fumar há 10 anos ou menos e 12,5\% não responderam essa questão. Ao serem questionados se tiveram recaídas após parar de fumar, 95,8\% dos entrevistados dentre os 24 exfumantes, responderam negativamente.

\section{Discussão}

Os dados do presente estudo permitiram a exploração do tema com vistas à ampliação para contingentes populacionais maiores, haja vista a importância de estudos abordando assunto considerado problema de saúde pública pela Organização Mundial de Saúde: uso de tabaco, especialmente entre trabalhadores.

A prevalência do consumo de tabaco encontrado neste estudo foi de $12,1 \%$, taxa essa menor do que a encontrada em estudo realizado na cidade de São Paulo, a qual computou $24 \%{ }^{(2)}$. Levando-se em consideração que a amostra foi composta por maioria do sexo feminino, $74,3 \%$ contra $26,7 \%$ do sexo masculino, era esperado que a prevalência do uso de tabaco nessa amostra fosse menor, comparando-se aos dados mencionados em estudos semelhantes $^{(19-20)}$.

Um dado importante de se observar é a elevada porcentagem de pessoas que nunca fumaram $(55,7 \%)$, assim como a encontrada em outros estudos cujos percentuais foram muito próximos ${ }^{(19-20)}$. 
Em relação aos problemas decorrentes do uso de tabaco, a maioria dos 13 entrevistados que responderam positivamente essa questão, declararam que sofreram problemas físicos decorrentes do uso da substância em questão, reafirmando os dados publicados em estudo semelhante ${ }^{(1)}$, no que se refere às doenças causadoras de morte devido ao hábito de fumar, como doenças cardiovasculares, cânceres, doenças respiratórias e outras.

Outro item relacionado aos problemas físicos, causados pelo uso do tabaco, refere-se aos motivos para parar de fumar declarados pelos fumantes desta amostra. Entre os que se consideraram fumantes, $88,8 \%$ gostariam de parar de fumar devido aos malefícios que o uso de tabaco ocasiona em sua saúde física. Da mesma forma, os ex-fumantes deste estudo tiveram o mesmo tipo de motivação para cessar o uso de tabaco. Tais achados indicam a importância de incluir essa temática nas ações educativas de prevenção, abordando os malefícios que o consumo de tabaco poderá acarretar na saúde tanto física quanto emocional e social do indivíduo.

$\mathrm{Na}$ amostra de fumantes deste estudo, 72,2\% deles já tentaram parar de fumar, mas continuam e as maiores dificuldades relatadas, no que tange à cessação do uso de tabaco, foram o hábito de fumar que possuem, a vontade que sentem em fumar, a ansiedade, o nervosismo, a falta de adaptação à medicação de apoio, os problemas familiares, o estado emocional e a influência de amigos e ambientes sociais. Esses dados corroboram estudos semelhantes, os quais apontam que a cessação definitiva do uso de tabaco ocorre, geralmente, após várias tentativas e a taxa de recaídas são elevadas ${ }^{(21)}$. Já a dificuldade em parar de fumar devido à influência de amigos e ambientes sociais refletem nas atitudes que reforçam positivamente a continuidade do vício pelos fumantes, encontrando, assim, dificuldades para cessar o hábito(22).

Entre a população que procurou ajuda para parar de fumar, obteve-se uma demonstração da existência de diversos métodos que auxiliam o alcance desse objetivo como uso de adesivos de nicotina, participação de terapias em grupo específica para parar de fumar e uso de medicações. Esses métodos são semelhantes aos encontrados em outro estudo sobre a temática ${ }^{(20)}$.

Dentre os ex-fumantes, 22 entrevistados pararam de fumar de uma vez e apenas 2 indivíduos diminuíram o número de cigarros até atingirem os seus objetivos. Essa amostragem pequena deve-se ao fato de o fumante relutar para procurar ajuda, bem como a condição de escassez de serviços especializados na identificação de pacientes de riscos, os quais se tornam barreiras para a procura por tratamento adequado $^{(2)}$.

\section{Considerações Finais}

O consumo de tabaco é considerado um dos maiores problemas de saúde pública já que seu uso encontra-se em segundo lugar nas pesquisas nacionais sobre o uso e abuso de substâncias psicoativas, atrás apenas do consumo abusivo de álcool. Tendo esse conhecimento, juntamente com os dados obtidos pelo presente estudo, destaca-se que a maioria dos fumantes anseia pela cessação do tabagismo, mas poucos o conseguem sem procurar por ajuda adequada, sendo necessário, dessa forma, fornecer apoio formal para o restante da população fumante para obtenção de êxito, tornando-se importante que os profissionais de saúde desenvolvam algum tipo de abordagem que seja efetiva junto aos fumantes. Deve-se, ainda, considerar que o sucesso para parar de fumar dependerá também da identificação dos fatores dificultadores envolvidos na cessação desse hábito.

Nos últimos 20 anos, a educação e a persuasão não foram suficientes para promover mudança política, cultural e social relacionada ao comportamento de fumar. As intervenções para interromper o uso de tabaco ainda não estão integradas às rotinas dos serviços de saúde no mundo.

Os resultados desta amostra de trabalhadores levase a refletir que o ambiente de trabalho possa atuar tanto como fator dificultador quanto facilitador do abandono e do início do hábito, considerando-se a convivência com outros fumantes e as tensões inerentes ao ambiente laboral. Assim, acredita-se na viabilidade de estudos que contribuam para o desenvolvimento de intervenções articuladas ao ambiente de trabalho, a fim de apoiar os funcionários com problemas relacionados ao uso de tabaco, identificar riscos e propor tratamento adequado aos mesmos.

\section{Referências}

1. CREMESP/AMB (BR). Usuários de substâncias psicoativas - abordagem, diagnóstico e tratamento. $2^{a}$.ed. São Paulo: Conselho Regional de Medicina do estado de São Paulo: Associação Médica Brasileira; 2003. 119 p.

2. Marques ACPR, Campana A, Gigliotti AP, Lourenço MTC, Ferreira MP, Laranjeira R. Consenso sobre o tratamento da dependência de nicotina. Rev Bras Psiquiatr. 2001;23(4): 200-14.

3. Pietrobon RC, Barbisan JN, Manfroi WC. Utilização do teste de dependência à nicotina de Fagerström como um instrumento de medida do grau de dependência. Rev Hosp Clín Porto Alegre. 2007;27(3):31-6.

4. Fagerström K. The epidemiology of smoking: health consequences and benefits of cessation. Drugs. 2002;62(2):1-9.

5. Ministério da Saúde (BR). Secretaria de Assistência à Saúde. Instituto Nacional de Câncer. Estimativas da Incidência e Mortalidade por Câncer. Rio de Janeiro (RJ): INCA; 2002.

6. Kaplan HI. Compêndio de psiquiatria: ciências do comportamento e psiquiatria clínica. $7^{\mathrm{a}}$ ed. Porto Alegre (RS): Artmed; 1997.

7. Ribeiro AS, Jardim JRB, Laranjeira RR, Alves AKS, Kesselring F, Fleissig L, et al. Prevalência de tabagismo na Universidade Federal de São Paulo, 1996 - dados preliminares de um programa institucional. Rev Assoc Med Bras. 1999;45(1):39-44.

8. Carrillo LPL, Mauro MYC. Uso e abuso de álcool e 
outras drogas: ações de promoção e prevenção no trabalho. Rev Enferm UERJ. 2003;11(1):25-33.

9. Associação Brasileira de Empresas de Pesquisa (ABEP) (BR). Critérios de Classificação Econômica Brasil; 2003. [acesso 15 ago 2007]. Disponível em http://www.abep. org/codigosguias/ABEP_CCEB.pdf.

10. Halty LS, Huttener MD, Netto ICO, SAntos VA, Martins G. Análise da utilização do Questionário de Tolerância de Fagerström (QTF) como medida da dependência de nicotina. J Pneumol. 2002;28(4):180-6.

11. Fagerström KO. Measuring degree of physical dependence to tobacco smoking with reference to individualization of treatment. Addict Behav. 1978;3(34):235-41.

12. Meneses-Gaya IC, Zuardi AW, Loureiro SR, Crippa JAS. As propriedades psicométricas do Teste de Fagerström para Dependência de Nicotina. J Bras Pneumol. 2009;35(1):73-82.

13. Castro MG, Oliveira MS, Moraes JFD, Miguel AC, Araujo RB et al. Qualidade de vida e gravidade da dependência de tabaco. Rev Psiq Clín. 2007;34(2):61-7.

14. Fagerstrom KO, Schneider NG. Measuring nicotine dependence: a review of the Fagerstrom Tolerance Questionnaire. J Behav Med. 1989;12(2):159-82.

15. Heatherton TF, Kozlowski LT, Frecker RC, Fagerström KO. The Fagerström Test for Nicotine Dependence: a revision of the Fagerström Tolerance Questionnaire. Br J Addict. 1991;86(9):1119-27.

16. Figlie NB, Pillon SC, Dunn J, Laranjeira R. The frequency of smoking and problem drinking among general hospital inpatients in Brazil - using the AUDIT and Fagerström questionnaires. Sao Paulo Med J. 2000;118(5):139-43.

17. Boni RD, Pechansky F. Prevalência de tabagismo em uma unidade de internação psiquiátrica. Rev Psiquiatr Rio Gd Sul. 2003;25(3):475-8.

18. Ministério da Saúde (BR). Conselho Nacional de Saúde. Resolução n. 196 de 10 de outubro de 1996: diretrizes e normas regulamentadoras de pesquisa envolvendo seres humanos. [acesso 17 set 2007]. Disponível em: www. conselho.saude.gov.br/Resolucoes/Reso196.doc.

19. Guazzelli AC, Terra M Filho, Fiss E. Tabagismo entre médicos da Região do ABC Paulista. J Bras Pneumol. 2005;31(6):516-22.
20. Sabry MOD, Sampaio HAC, Silva MGC. Tabagismo e etilismo entre funcionários da Universidade Estadual do Ceará. J Bras Pneumol. 1999;25(6):313-20.

21. Chatkin JM. A influência da genética na dependência tabágica e o papel da farmacogenética no tratamento do tabagismo. J Bras Pneumol. 2006;32(6):573-9.

22. Araujo AJ, Menezes AMB, Dorea AJPS, et al. Diretrizes para cessação do tabagismo. J Bras Pneumol. 2004;30, supl 2, (2):S1-S76.

\section{Como citar este artigo:}

Gherardi-Donato ECS, Lopes M, Santiago MR, Corradi-Webster CM. Caracterização de consumo e dependência de tabaco entre trabalhadores de uma instituição de nível superior. SMAD, Rev. Eletrônica Saúde Mental Álcool Drog. (Ed. port.). set.-dez. 2011 [acesso: $\_-1$ ] $; 7(3): 155-60$. Disponível em: 